\title{
Os Efeitos do Jejum Intermitente nos Parâmetros Bioquímicos de Ratos Wistar
}

\section{The Effects of Intermittent Fasting in the Wistar Rats' Biochemical Parameters}

André Luiz Jacques Chamorroa; Beatriz Kiyoko Fujii ; Eduardo Machado Bacha'; Gabriel Cabral Valadares Lins; Kawana Ribeiro Yamakawa ${ }^{a}$; Leda Márcia Araújo Bentoa; Luiza Engel Lorenzonª Maethany Nayandra Oliveira da Silva ${ }^{\mathrm{a}}$; Rafaela Alvez Lima ${ }^{\mathrm{a}}$; Victor Yukio Iwata Seraphimaa; William Barbosa Martins Asato ${ }^{\mathrm{b}}$

aUniversidade Anhanguera-Uniderp. MS, Brasil.

bUniversidade Estadual de Mato Grosso do Sul, MS, Brasil. *E-mail:

\section{Resumo}

O presente estudo teve como objetivo avaliar as repercussões bioquímicas em ratos Wistar submetidos ao jejum intermitente por 15,30 e 60 dias, modelo de dieta intercalada por períodos de restrição total de alimentação com períodos de alimentação ad libitum. O estudo foi realizado com 40 ratos Wistar, machos divididos em três grupos, todos recebendo a ração Nuvital ${ }^{\circ}$. Os ratos submetidos ao jejum intermitente tiveram esse tipo de dieta durante 3 dias na semana e nos demais dias tiveram acesso livre a ração e água. Os animais foram pesados semanalmente e, após o período de jejum intermitente, foram eutanasiados para a coleta de sangue e análise bioquímica (glicemia, triglicérides, colesterol total e frações). Houve redução significativa do peso e triglicerídeos nos animais do grupo de jejum em relação ao grupo controle durante 15 dias. Em relação ao grupo de 30 dias de jejum, houve redução significativa de colesterol total e HDL. Nos animais submetidos à dieta durante 60 dias, houve diminuição significativa de colesterol total e HDL, no entanto, o consumo de água aumentou consideravelmente. Conclui-se que o jejum intermitente durante 15 dias causou alterações bioquímicas favoráveis ao metabolismo desses animais.

Palavras-chave: Jejum Intermitente. Restrição Calórica. Prevenção.

\begin{abstract}
This experiment was conducted to evaluate the biochemical effects in healthy Wistar rats submitted by 15, 30 and 60 days to intermittent fasting, a model of interspersed diet between periods of total food restriction withfood ad libitum period. The study was performed with 40 male Wistar rats randomly divided into 3 groups, all receiving Nuvital $\mathbb{~}$. The rats submitted to intermittent fasting had this type of diet during 3 days a week and on the other days had free access to food. They received water ad libitum every day. The analysis related to metabolic changes resulting from fasting were performed by means of serum biochemical analysis (blood glucose, triglycerides, total cholesterol and HDL) at the end of the experiment. There was a significant reduction of weight and triglycerides from the fasting group in relation to the control group in 15 days, there was no significant increase in parameters during this period of diet. Concerning the 30-day fasting group, there was a significant reduction of total cholesterol and HDL. Therefore, Intermittent Fasting in 15 days only demonstrated biochemical benefits.
\end{abstract}

Keywords: Intermittent Fasting. Caloric restriction. Prevention.

\section{Introdução}

A qualidade de vida e a busca pela manutenção da saúde adquiriram grande destaque na literatura atual. Novas formas para combater os possíveis aumentos no índice da obesidade e síndromes metabólicas na população, buscando uma vida saudável, têm sido estudadas. Entre as quais há o Jejum Intermitente (JI), que se caracteriza por períodos de alimentação livre intercalados com períodos de restrição de ingesta calórica (HARVIE; HOWELL, 2017).

É sabido que a prática do jejum intermitente é milenar e os primeiros achados datam de 600 mil a.C. a 25 mil a.C., período histórico no qual o jejum era fruto da incerteza de quando ocorreria a próxima alimentação, uma vez que o alimento era obtido por meio da caça (AZEVEDO et al., 2013). Nesse contexto, o jejum intermitente ocorria de forma involuntária. Nos dias atuais, a prática voluntária da restrição de ingesta calórica está retornando à rotina de milhares de pessoas como forma de amenizar os impactos causados pelo estilo de vida e rotina alimentar não saudável das sociedades modernas.

Foi verificada uma escassez de experimentos ligados ao Jejum Intermitente (JI) em indivíduos sem comorbidades metabólicas e/ou obesidade, e seus resultados bioquímicos e ponderais seriados em períodos de tempo prolongado. Os benefícios do Jejum Intermitente (JI) resultaram em perda de peso e redução da massa gorda, além de toda ação cardioprotetora pela melhora da pressão sanguínea e da frequência cardíaca. Também houve melhorias no perfil lipídico, com aumento de HDL e diminuição do colesterol total e LDL (AZEVEDO et al., 2013).

Além disso, há relatos da perda de peso com manutenção de massa magra, após Jejum Intermitente (JI) de quatro semanas (normolipídica) com camundongos obesos, induzidos por dieta hiperlipídica de oito semanas (GOTTHARDT et al., 2016). Nas primeiras 24 horas do jejum, há utilização das reservas de glicose do organismo. Após esse período, são utilizadas as reservas lipídicas para obter energia pela gliconeogênese com formação de corpos cetônicos, ocasionando perda 
de peso (SOETERS et al., 2009). Essa transição decorre, principalmente, pela ação dos hormônios glucagon, cortisol, GH e catecolaminas (RONA, 2017).

A restrição energética atinge principalmente a reserva de gordura visceral (abdominal, hepática e intramuscular), pois esta é mais sensível a ação das catecolaminas (HARVIE; HOWELL, 2017). Entretanto, períodos prolongados de jejum (de 1 a 2 dias) elevam o nível de ácidos graxos livres quando comparados ao jejum contínuo de 12 horas durante a noite, ocasionando resistência à insulina e acúmulo de triglicérides intramiocelulares nos músculos esqueléticos em humanos.

A peroxidação lipídica provinda da degradação oxidativa produz radicais livres que são citotóxicos. O Ramadã aumenta o estresse oxidativo, levando a danos celulares maiores e ganho de peso (BAHAMMAM et al., 2016). Em seu posterior estudo, obtiveram resultados diferentes: não houve alterações do fator MDA (marcador de estresse oxidativo) nos homens submetidos ao estudo durante o mesmo período. A diferença é que no segundo houve controle de horas de sono, atividade física, duração do jejum, uso de medicamentos, fumo e composição das refeições. Tudo isso, de acordo com os autores, tem influência nos níveis de MDA. Houve melhora no perfil glicêmico de homens saudáveis submetidos a 60 horas de jejum, quando foi observada uma queda de $30 \%$ na glicose plasmática, de 50\% na insulina e um aumento na sensibilidade à insulina (AZEVEDO et al., 2013). Além disso, no caso de homens saudáveis submetidos a ciclos de alimento e restrição, muda-se o status metabólico para melhor: foi o primeiro estudo em humanos em que foi possível obter uma aumentada ação da insulina na captação sistêmica de glicose, além da lipólise de tecido adiposo, por meio do jejum intermitente (HALBERG et al., 2005).

Tais ciclos são importantes como iniciadores dos genes que levam a melhorias na função metabólica. Até mesmo um jejum intermitente prolongado em que os indivíduos se submeteram a um regime completo no início dos estudos obteve alterações mantidas durante toda a pesquisa.

Sendo assim, o presente estudo avaliou as alterações bioquímicas da prática do jejum intermitente em ratos machos Wistar, em um curto, médio e longo prazo, no que diz respeito aos perfis lipídico e glicêmico, além de comparar o consumo alimentar e hídrico e alterações ponderais dos animais submetidos à dieta comparados aos animais com controle em dieta livre (Ad libitum) em 15, 30 e 60 dias de experimento. Os objetivos do presente trabalho foram analisar a segurança e eficácia do JI no metabolismo dos animais avaliados para possível inferência em pesquisas em humanos.

\section{Material e Métodos}

Mediante o parecer n ${ }^{\circ} 3048$ da Comissão de Ética no Uso de Animais da Anhanguera Educacional, para este estudo foram usados 40 ratos da linhagem Wistar (Rattus novergicus albinus), machos, adultos, com o peso variando de $250 \mathrm{~g}$ a 300g, provenientes do Biotério da Universidade Federal do Mato Grosso do Sul e mantidos durante o estudo no Biotério de manutenção da Universidade Anhanguera Uniderp (Unidade Agrárias), na cidade de Campo Grande, Mato Grosso do Sul.

Para aferição da glicemia foi retirada uma gota de sangue da veia da cauda colocando sobre fitas reagentes da marca ACCU CHEC. Os animais foram mantidos em condições controladas de luminosidade (12 horas de luz/12 horas de escuro) e temperatura (média de $23^{\circ} \mathrm{C}$ ), com livre acesso a água e ração comercial ou com livre acesso a água e 8 horas de acesso a ração comercial Nuvital ${ }^{\circledR}$.

Os ratos foram divididos em três grandes grupos, sendo esses: Grupo A (4 animais submetidos ao jejum e 4 ratos como grupo controle) com duração de 15 dias; Grupo B (8 animais submetidos ao jejum e 8 ratos como grupo controle) com duração de 30 dias; e Grupo C (8 animais submetidos ao jejum e 8 ratos como grupo controle) com duração de 60 dias. Os animais do grupo controle (GCO) receberam dieta padrão composta por Nuvital ${ }^{\circledR}$ ad libitum, assim como a ingestão de água; no grupo jejum intermitente (GJI), a ração esteve disponível por somente oito horas durante três dias na semana (período noturno, devido ao ciclo circadiano invertido dos ratos), sendo que nos outros dias a ingesta hídrica e alimentar foi ad libitum.

Os animais dos grupos controle e jejum intermitente foram ambientados antes do início do protocolo do estudo, isto é, foram mantidos nas condições descritas acima por cinco dias consecutivos. Entretanto, esses dias de ambientação foram considerados para a contagem do tempo de jejum. O controle de peso por meio da balança foi realizado semanalmente em todos os grupos do estudo, enquanto o consumo de ração e água foi quantificado durante três dias fixos na semana.

Ao término do período experimental de cada grupo, a eutanásia foi realizada por administração via intraperitoneal de dose letal de anestésico cloridrato de Ketamina + Xilazina (0,4 $\mathrm{ml}$ a cada 100g) (MASSONE, 2003). A técnica empregada de dose letal já está bastante documentada e aceita pelos comitês de ética e está de acordo com o Anexo à Resolução $n^{0} 13$, de 20 de setembro de 2013 (BRASIL, 2013).

Após a eutanásia, foi realizada a retirada dos fluidos (sangue) e dos tecidos (fígado, rim e aorta), e os animais foram congelados em freezer a $-30{ }^{\circ} \mathrm{C}$, sendo no dia de coleta postos em um contêiner para descarte de dejetos localizado na Universidade Uniderp, campus Agrárias. A empresa Spielmann \& Spielmann LTDA (Atitude), localizada na Avenida Engenheiro Annes Salim Saad, $n^{\circ}$ 479, Lote 17 - Quadra 12, Bairro Polo Empresarial, Campo Grande (Mato Grosso do Sul), foi responsável pelo recolhimento e incineração do material.

O perfil lipídico foi avaliado através da dosagem de colesterol total, HDL e triglicerídeos, utilizando os soros dos animais dos grupos citados acima. As análises foram realizadas utilizando kits comerciais Gold Analisa e no equipamento 
Bioplus 200. As análises das dosagens bioquímicas foram o teste " $\mathrm{t}$ " student. Os valores de $\mathrm{p}<0,05$ foram considerados significativos e o intervalo de confiança é de $95 \%$.

O coeficiente de eficácia alimentar (CEA) de todos os grupos foi avaliado utilizando o peso inicial e final dos animais e a quantidade de ração ingerida. O CEA é a relação do ganho de massa e da quantidade de alimento ingerido no período avaliado através da fórmula: PF-PI/TA. PF, PI e TA são, respectivamente, peso final, peso inicial e quantidade total de alimento consumido em gramas (NERY et al., 2011). Sendo assim, quanto maior for o valor do CEA, menor será a eficácia alimentar e vice-versa.

\section{Resultados e Discussão}

\subsection{Consumo de água e ração}

O grupo submetido ao Jejum Intermitente (JI) pelo período de 15 dias obteve um coeficiente de eficácia alimentar (CEA) $17,98 \%$ menor quando comparado ao grupo controle avaliado no mesmo período. Nos grupos de 30 e 60 dias ocorreu um aumento no CEA, sendo de $4 \%$ no grupo submetido a 30 dias de experimento e de $12,5 \%$ no grupo submetido a 60 dias (Quadro 1).

Quadro 1 - Avaliação dos efeitos do jejum intermitente nos valores de ingesta hídrica e alimentar

\begin{tabular}{|c|c|c|c|c|c|c|}
\hline Variáveis: & $\begin{array}{c}\text { CO } \\
\text { 15d }\end{array}$ & $\begin{array}{c}\text { JI } \\
\text { 15d }\end{array}$ & $\begin{array}{c}\text { CO } \\
\mathbf{3 0 d}\end{array}$ & $\begin{array}{c}\text { JI } \\
\mathbf{3 0 d}\end{array}$ & $\begin{array}{c}\text { CO } \\
\mathbf{6 0 d}\end{array}$ & $\begin{array}{c}\text { JI } \\
\mathbf{6 0 d}\end{array}$ \\
\hline $\begin{array}{c}\text { Ingesta } \\
\text { Hídrica } \\
\text { (ml) }\end{array}$ & 156,9 & 155,51 & 153,51 & 153,33 & 149,79 & $162,33 *$ \\
\hline $\begin{array}{c}\text { Ingesta } \\
\text { alimentar } \\
\text { (g) }\end{array}$ & 84,71 & 99,09 & 129,06 & 124,22 & 141,1 & 148,31 \\
\hline CEA & 1,87 & 1,54 & 0,9 & 0,96 & 1,08 & 1,15 \\
\hline
\end{tabular}

CO: Grupo Controle; JI: Grupo Jejum Intermitente; $\mathrm{d}: \mathrm{n}^{\circ}$ dias.

Fonte: Dados da pesquisa.

No GCO de 15 dias, o CEA foi de 1,87; enquanto no GJI de 15 dias foi de 1,54, o que indica que o GJI nesse período ingeriu mais alimento e ganhou menos peso em comparação ao grupo controle 15 dias. Já no GCO de 30 dias, o CEA foi de 0,9. Em contrapartida, no GJI 30 dias foi de 0,96, o que indica que o GJI nesse período ingeriu menos alimento e ganhou menos peso em comparação ao grupo controle 30 dias (Quadro 1). No GCO de 60 dias, o CEA foi de 1,08; ao passo que no GJI 60 dias foi de 1,15, o que indica que o GJI nesse período ingeriu mais alimento e ganhou menos peso em comparação ao grupo controle 60 dias (Quadro 1).

Portanto, o grupo submetido ao jejum intermitente por 15 dias mostrou um aumento do consumo médio alimentar em $14,3 \mathrm{~g}$, associado ao aumento do ganho ponderal em 3,9\%, quando comparado ao grupo controle 15 dias. Uma hipótese para tal explicação seria uma possível tentativa em compensar o período de restrição alimentar nos períodos ad libitum, ou seja, os animais consumiriam uma maior quantidade de ração, levando ao maior ganho ponderal.
Entretanto, nos grupos de 30 dias o grupo jejum consumiu $4,84 \mathrm{~g}$ a menos que o grupo controle, ambos em 30 dias, associado ao ganho ponderal de $45 \mathrm{~g}$ a menos quando comparado ao grupo controle no mesmo período (Quadro 1).

Já o GJI 60 dias ingeriu 7,21 g a mais que o GCO 60 dias, quando comparadas as médias de consumo de ração. Ao compararmos as médias ponderais, houve ganho de $1,1 \mathrm{~g}$ a menos no GJI de 60 dias em relação ao GCO 60 dias (Quadro 1).

Em relação ao consumo de água, não foi observada uma grande diferença na ingesta entre os grupos GJI 15 dias e GCO 15 dias e GJI 30 dias e GCO 30 dias (p>0,05), revelando que a prática do jejum por tais períodos não causou alterações significativas na ingesta de água. Já no grupo jejum de 60 dias, houve aumento significativo $(\mathrm{p}<0,05)$ de $8,82 \mathrm{ml}$ na ingesta média de água, quando comparado ao grupo controle 60 dias (Quadro 1).

\subsection{Avaliação Ponderal}

Neste presente trabalho, diferentemente do ocorrido no estudo feito por Chausse et al. (2014) e Moraes (2016), a média aritmética do peso médio por animal aumentou 15,3 g (3,9\% do peso corporal) na comparação entre o grupo controle e jejum de 15 dias, porém não houve diferença significante ( $>>0,05)$ (Quadro 2).

Quando 2 - Avaliação dos efeitos do jejum intermitente nos valores de peso

\begin{tabular}{|c|c|c|c|c|c|c|}
\hline Variáveis: & $\begin{array}{c}\text { CO } \\
\text { 15d }\end{array}$ & $\begin{array}{c}\text { JI } \\
\text { 15d }\end{array}$ & $\begin{array}{c}\text { CO } \\
\text { 30d }\end{array}$ & $\begin{array}{c}\text { JI } \\
\text { 30d }\end{array}$ & $\begin{array}{c}\text { CO } \\
\text { 60d }\end{array}$ & $\begin{array}{c}\text { JI } \\
\text { 60d }\end{array}$ \\
\hline Peso (g) & 377 & $392,33^{*}$ & 381,4 & 336 & 408,35 & 407,25 \\
\hline
\end{tabular}

Fonte: Dados da pesquisa.

Já com 30 dias de jejum, houve uma diminuição de 45,4 g no peso médio, comparado ao grupo controle, com redução significativa do peso corporal $(\mathrm{p}<0,05)$. Ao comparamos os grupos de 60 dias, a variação entre os grupos foi menor que $1,1 \mathrm{~g}$ (menos de 1\%), sendo que não houve uma diferença estatística significativa ( $\mathrm{p}>0,05)$ (Quadro 2).

Segundo vários autores, como Sakamoto e Grunewald (1987), Santos (2017), Harvie et al. (2014, 2011) e Klempel et al. (2010), o JI apresentou diminuição no peso, quando comparado ao grupo com dieta ad libitum, tanto em humanos quanto em animais, não só em indivíduos obesos, como também nos eutróficos. O estudo de Chausse et al. (2014) apontou para uma diminuição média de 27,8 g na avaliação ponderal, quando comparado um grupo de animais submetidos ao JI por três semanas ao grupo controle, sendo essa diminuição classificada pelos autores como significativa. Isso também aconteceu no estudo de Moraes (2016), em que o grupo submetido ao JI resultou em diminuição do peso corporal a partir de duas semanas.

É possível compreender as hipóteses geradas por Moraes (2016) e Klop et al. (2013) do aumento gasto energético 
basal como resultante ao JI, principalmente pelo metabolismo lipídico, quando vemos o aumento do gasto energético. Em curto prazo (15 dias), aumentou-se o gasto energético (com maior eficiência alimentar), assim como a ingestão e consequentemente o peso podem ter pequenas variações positivas.

Com uma maior adaptação do organismo (30 dias), o gasto energético total provavelmente continuou elevado, porém com uma ingestão alimentar muito parecida com o grupo controle, o que resultou numa perda de peso. Os períodos maiores de jejum (60 dias) mostram que os possíveis gastos energéticos são reequilibrados, de maneira que a ingestão e a eficiência alimentar, assim como o peso corporal, sejam semelhantes ao grupo controle, retornando à homeostasia.

Em suma, quando praticado por 15 dias, o jejum intermitente contribuiu para a redução da massa corporal nos ratos, aumentando a eficiência alimentar. Ao prazo de 30 e 60 dias, a eficiência alimentar não mostrou alteração significativa nos grupos submetidos ao jejum intermitente.

\subsection{Avaliação do Perfil Lipídico}

\subsubsection{Colesterol total}

As diferenças de concentrações séricas de colesterol total não foram estatisticamente significantes entre os grupos controle e JI de 15 dias. Entretanto, no grupo JI 30 dias foi observada uma redução significativa quando comparado ao controle 30 dias. O grupo JI 60 dias apresentou redução significativa em relação ao grupo controle 60 dias (Figura 1).

Figura 1 - Avaliação dos efeitos do jejum intermitente nos níveis de colesterol. (Análise Estatística: teste t student)

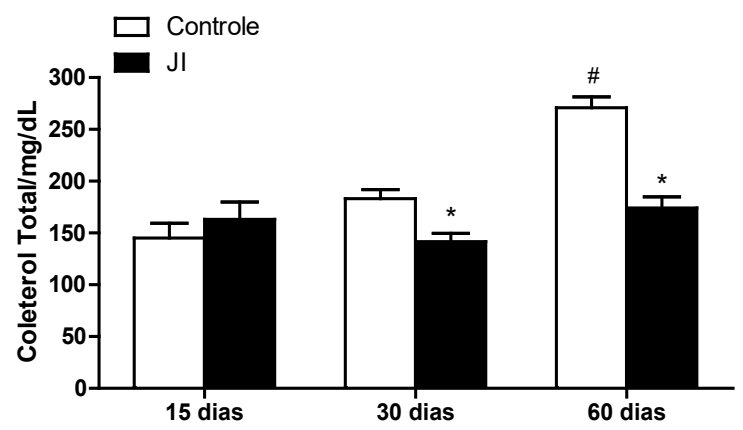

A concentração sérica de colesterol foi avaliada nos grupos controle e jejum intermitente, com ensaios utilizando kits comerciais Gold Analisa. Os resultados são expressos como média \pm EPM de unidades/litro de soro $(\mathrm{n}=4$ a 8$) .{ }^{*} \mathrm{p}<0,05$ quando comparado entre o grupo controle 15 e 30 dias e o controle 60 dias.

Fonte: Dados da pesquisa.

O perfil lipídico mostrou alteração no marcador HDL no jejum intermitente em dias alternados, não havendo alteração dos demais parâmetros como de LDL, triglicérides e colesterol total (TREPANOWSKI et al., 2017). Essa alteração lipídica seria resultado de um déficit energético e/ou por perda de peso (KLOP et al., 2013).

\subsubsection{HDL}

No presente estudo, os valores séricos de HDL no grupo JI 15 dias não apresentaram alteração significativa quando comparados ao controle de mesmo período. Nos grupos JI de 30 e 60 dias foi observada uma diminuição significativa, se comparados aos grupos controle de 30 e 60 dias (Figura 2).

Figura 2 - Avaliação dos efeitos do jejum intermitente nos níveis de HDL

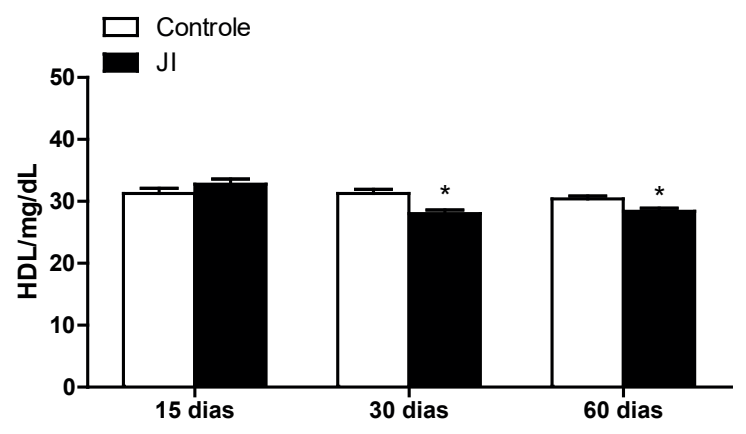

A concentração sérica de HDL foi avaliada nos grupos controle e jejum intermitente, com ensaios utilizando kits comerciais Gold Analisa. Os resultados são expressos como média \pm EPM de unidades/litro de soro $(\mathrm{n}=4$ a 8$) .{ }^{*} \mathrm{p}<0,05$ quando comparado ao respectivo grupo controle. (Análise Estatística: teste t student).

Fonte: Dados da pesquisa.

A diminuição de HDL observada nos grupos de 30 e 60 dias deve-se à necessidade de diminuição de, pelo menos, $5 \%$ do peso para melhora do perfil lipídico, fato que não foi constatado neste estudo. O jejum intermitente é benéfico ao normalizar os índices lipídicos de indivíduos obesos e dislipidêmicos, tendo pouca influência em indivíduos saudáveis, como ocorrido no presente estudo. Outra hipótese apresentada é de que 16 horas de jejum seriam muito pouco para alterar os valores de colesterol (GABEL et al., 2018).

Comparando as medidas das frações do colesterol do mesmo grupo, antes e após o jejum, foi observada a diminuição do LDL em até $13 \mathrm{mg} / \mathrm{dl}$, elevação do HDL em até $5 \mathrm{mg} / \mathrm{dl}$ e diminuição do colesterol total em $9 \mathrm{mg} / \mathrm{dl} \mathrm{em}$ indivíduos com síndrome metabólica (SANTOS; MACEDO, 2018). De acordo com os autores, em outra análise de estudo realizado com indivíduos do sexo masculino durante o Ramadan (jejum diário de 12 horas por 30 dias), foi observado um aumento da Apolipoproteína A-1 no sangue, precursor do HDL, aumentando os níveis séricos do mesmo (IBRAHIM et $a l .$, 2008). Ainda em sua revisão, em indivíduos adultos (2550 anos) do sexo masculino foi observada uma diminuição dos níveis séricos de Apolipoproteína B, precursora do LDL, justificando a redução deste marcador com o jejum intermitente.

Em comparação, na maior parte dos artigos analisados por Santos e Macedo (2018), realizando 12 horas de jejum por 30 dias em indivíduos saudáveis, não houve alteração significativa dos níveis de HDL e LDL, apesar de haver aumento do HDL em poucos trabalhos analisados. Em um estudo analisado com indivíduos do sexo masculino durante o Ramadan (30 dias 
de jejum de 12 horas), foi observada a redução dos níveis de LDL e o aumento de HDL, comparando os indivíduos antes e no final do período. Tal resultado é explicado pelos autores através da diminuição da ingesta calórica e lipídica, que seria responsável por diminuir a produção dessas moléculas pelo organismo (SANTOS; MACEDO, 2018).

\subsubsection{Triglicerídeos}

O nível de triglicerídeos no grupo JI 15 dias reduziu significativamente em relação ao grupo controle de 15 dias. Não houve alteração significativa nos grupos de 30 e 60 dias.

Figura 3 - Avaliação dos efeitos do jejum intermitente nos níveis de triglicerídeos

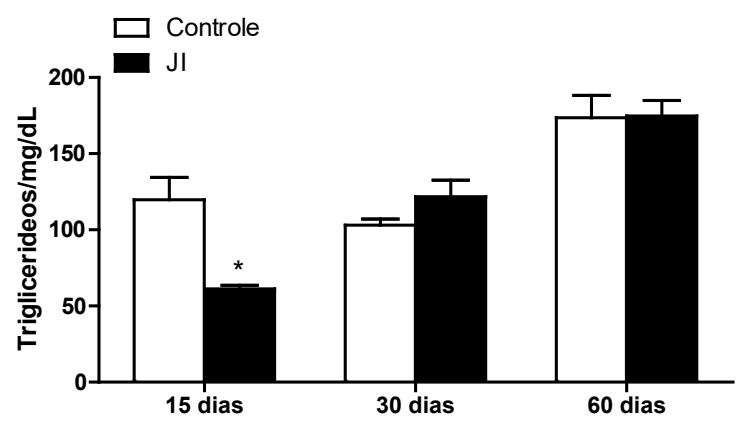

A concentração sérica de triglicerídeos foi avaliada nos grupos controle e jejum intermitente, com ensaios utilizando kits comerciais Gold Analisa. Os resultados são expressos como média \pm EPM de unidades/litro de soro $(\mathrm{n}=4$ a 8$) . * \mathrm{p}<0,05$ quando comparado ao respectivo grupo controle. (Análise Estatística: teste $t$ student).

Fonte: Dados da pesquisa.

Comparando os grupos JI e controle 15 dias, observamos redução significativa dos valores de triglicérides. Comparando os grupos JI e controle 30 e 60 dias, foi observado um aumento não significativo dos valores de triglicérides.

O JI causa a oscilação do metabolismo de triglicerídeos entre o anabolismo (gliconeogênese e lipogênese novamente) e catabolismo (lipólise). Essa lipólise decorre da mobilização dos triglicerídeos para substrato de energias para o organismo (AZEVEDO et al., 2013).

A melhora do perfil lipídico no JI (lipoproteínas, colesterol e triglicérides séricos) ocorre pela perda de massa gorda (SANTOS; MACEDO, 2018). Há redução da massa gorda, colesterol total, LDL e concentrações de triglicerídeos em seu estudo de jejum em dias alternados realizado com mulheres por 8 semanas (VARADY et al., 2015).

Outro estudo com humanos apresentou que houve um aumento significativo no LDL e diminuição no HDL. Parece que o efeito do jejum sobre os níveis séricos de lipídios pode estar relacionado à dieta nutricional ou à resposta bioquímica à fome (ZIAEE et al., 2006).

Em mais um estudo, não houve diferença significativa no colesterol total, LDL, HDL e triglicerídeos nos grupos que realizaram jejum intermitente em dias alternados, nos quais camundongos foram usados por 12 meses (YANG et al., 2016).

\subsection{Avaliação do Perfil Glicêmico}

\subsubsection{Glicose}

Figura 4 - Avaliação dos efeitos do jejum intermitente nos níveis de glicose

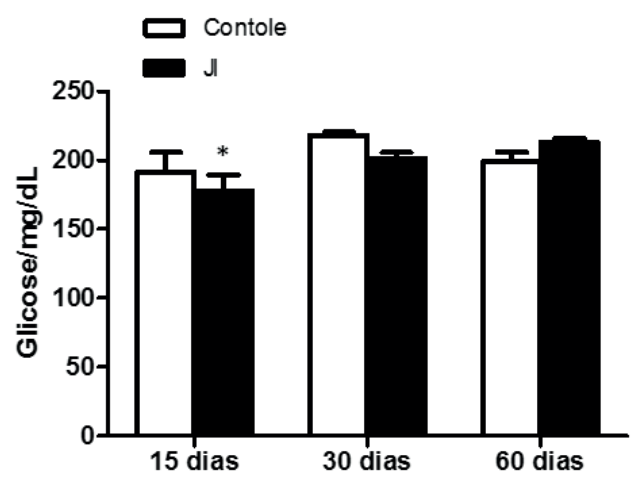

A concentração sérica de glicose foi avaliada nos grupos controle e jejum intermitente, com ensaios utilizando kits comerciais Gold Analisa. Os resultados são expressos como média \pm EPM de unidades/litro de soro $(\mathrm{n}=4$ a 8). (Análise Estatística: teste $\mathrm{t}$ student).

Fonte: Dados da pesquisa.

$\mathrm{Na}$ literatura, não há consenso a respeito dos efeitos do jejum intermitente sobre os índices glicêmicos basais e pósprandiais a curto, médio e longo prazo. Em pesquisa com 16 humanos submetidos a 12 horas diárias de JI pelo período de 22 dias não ocorreram alterações significantes na glicemia do grupo estudado em relação ao grupo controle (HEILBRONN et al., 2005). Uma pesquisa com ratos submetidos ao jejum intermitente por 24 horas duas vezes na semana, com dieta hiperglicemiante no restante dos dias a fim de verificar os efeitos do jejum intermitente na redução da incidência de diabetes a longo prazo, mostrou que houve redução dos níveis de glicose sérica nos ratos submetidos ao JI (AZEVEDO et al., 2013).

Em comparação entre resultados glicêmicos de ratos submetidos ao Jejum Intermitente de 10 horas diárias e dieta de restrição calórica a curto prazo (4 semanas) e a longo prazo (32 semanas), mostraram que a curto prazo não houve alteração significativa dos níveis glicêmicos nos ratos submetidos ao jejum intermitente, enquanto que a longo prazo houve importante intolerância à glicose, com aumento dos índices glicêmicos nos ratos submetidos ao JI (CERQUEIRA et al., 2011).

Portanto, no presente trabalho, a média dos resultados glicêmicos dos grupos de ratos submetidos ao JI a curto e médio prazo (15 e 30 dias) foi semelhante aos resultados relatados por Azevedo et al. (2013), demonstrando redução da glicemia. Isso ocorre provavelmente pela maior eficiência do efeito da insulina circulante após longos períodos de jejum, levando a um cenário de condições hipoglicemiantes.

Em relação aos efeitos do JI em longo prazo (60 dias), os resultados do presente trabalho se assemelham ao estudo de Cerqueira et al. (2011), evidenciando aumento dos índices glicêmicos nos ratos submetidos ao JI, possivelmente 
pelo fenômeno de intolerância à glicose, decorrente da hipoestimulação pancreática para produção e liberação insulínica regular.

\section{Conclusão}

Após a observação de ratos Wistar e análise dos dados significativos após 15, 30 e 60 dias submetidos ao jejum intermitente (JI), concluímos que esta prática causou alterações benéficas à saúde dos animas como a redução do colesterol. Baseado nos resultados, concluímos que a opção mais viável é a de 15 dias de JI em ratos, visto que períodos superiores causaram prejuízos do perfil lipídico e glicêmico aos animais. Desta forma, consideramos que o jejum intermitente a longo prazo possivelmente traz efeitos prejudiciais, não suprindo o princípio da beneficência.

Sugerimos para as futuras pesquisas, a realização do JI por um período maior de tempo e com maior frequência, por promover maiores benefícios de acordo com a literatura. É relevante a dosagem de hormônios influentes do JI (insulina, glucagon e cortisol) e dos parâmetros antes e depois de realizar o jejum. Além disso, é interessante fazer a identificação dos animais durante o estudo para uma melhor comparação entre grupos e indivíduos.

\section{Referências}

AZEVEDO, F.R.; IKEOKA, D.; CARAMELLI, B. Effects of intermittent fasting on metabolism in men. Rev. Assoc. Med. Bras., v.59, n.2, p.167-173, 2013. doi:10.1016/j.ramb.2012.09.003

BAHAMMAM, A.S.; PANDI-PERUMAL, S.R.; ALZOGHAIBI, M. A. The effect of Ramadan intermittent fasting on lipid peroxidation in healthy young men while controlling for diet and sleep: a pilot study. Ann. Thor. Med., v.11, n.1, p.43-48, 2016. doi: 10.4103/1817-1737.172296

BRASIL. Resolução normativa $n^{\circ} 13$ de 20 de setembro de 2013. Baixa as Diretrizes da Prática de Eutanásia do Conselho Nacional de Controle de Experimentação Animal - Concea. Ministério da Ciência, Tecnologia e Inovação. Brasília: Concea, 2013.

CERQUEIRA, F.M. et al. Long-term intermittent feeding, but not caloric restriction, leads to redox imbalance, insulin receptor nitration, and glucose intolerance. Free Rad. Biol. Med., v.51, n.7, p.1454-1460, 2011. doi: 10.1016/j.freeradbiomed.2011.07.006

CHAUSSE, B. et al. Intermittent fasting induces hypothalamic modifications resulting in low feeding efficiency, low body mass and overeating. Endocrinol., v.155, n.7, p.2456-2466, 2014. doi: 10.1210/en.2013-2057

GABEL, K. et al. Effects of 8-hour time restricted feeding on body weight and metabolic disease risk factors in obese adults: a pilot study. Nutr. Healthy Aging, v.4, n.4, p.345-353, 2018. doi: 10.3233/NHA-170036

GOTTHARDT, J.D. et al. Intermittent fasting promotes fat loss with lean mass retention, increased hypothalamic norepinephrine content, and increased neuropeptide y gene expression in dietinduced obese male mice. Endocrinology, v.157, n.2, p.679-691, 2016. doi: 10.1210/en.2015-1622

HALBERG, N. et al. Effect of intermittent fasting and refeeding on insulin action in healthy men. J. Appl. Physiol., v.99, n.6, p.2128-2136, 2005. doi: 10.1152/japplphysiol.00683.2005

HARVIE, M.; HOWELL, A. Potential benefits and harms of intermittent energy restriction and intermittent fasting amongst obese, overweight and normal weight subjects - a narrative review of human and animal evidence. J. Behavioral Scie., v.7, n.1, p. 4, 2017. doi: 10.3390/bs7010004

HEILBRONN, L.K. et al. Alternate-day fasting in nonobese subjects: effects on body weight, body composition, and energy metabolism. Am. J. Clin. Nutr., v.81, n.1, p.69-73, 2005. doi: 10.1093/ajen/81.1.69

IBRAHIM, W.H. et al. Effect of ramadan fasting on markers of oxidative stress and serum biochemical markers of cellular damage in healthy subjects. Ann. Nutr. Metabol., v.53, n.3/4, p.175-181, 2008. doi: 10.1159/000172979

KLEMPEL, M.C. et al. Dietary and physical activity adaptations to alternate day modified fasting: implications for optimal weight loss. Nutr. J., v.9, p.35, 2010. doi: 10.1186/1475-2891-9-35.

KLOP, B.; CABEZAS, J.W.F.E.; CASTRO, M. Dyslipidemia in obesity: mechanisms and potential targets. Nutrients, v.5, n.4, p.1218-1240, 2013. doi: 10.3390/nu5041218

MASSONE, F. Atlas de anestesiologia veterinária. São Paulo: Roca, 2003.

MORAES, R.C.M. Impactos de uma estratégia de jejum intermitente associada a treinamento de endurance na composição corporal e desempenho físico de ratos Wistar. Uberaba: Universidade do Triângulo Mineiro, 2016.

NERY, C.S. et al. Medidas murinométricas e eficiência alimentar em ratos provenientes de ninhadas reduzidas na lactação e submetidos ou não ao exercício de natação. Rev. Bras. Med. Esporte, v.17, n.1, p.49-55, 2011. doi: 10.1590/S151786922011000100010

RONA, A. Metabolic effects of intermittent fasting. Department of Nutritional Sciences Faculty of Health and Medical Sciences. Universidade de Surrey, 2017.

SAKAMOTO, K.; GRUNEWALD, K.K. Beneficial effects of exercise on growth of rats during intermittent fasting. J. Nutr., v.117, n.2, p.390-395, 1987. doi: 10.1093/jn/117.2.390

SANTOS, H.O.; MACEDO, R.C.O. Impact of intermittent fasting on the lipid profile: assessment associated with diet and weight loss. Clin. Nutr. Euro. Soc. Clin. Nutr. Metabol., v.24, p.14-21, 2018. doi: 10.1016/j.clnesp.2018.01.002.

SOETERS, M.R. et al. Intermittent fasting does not affect wholebody glucose, lipid, or protein metabolism. Am. J. Clin. Nutr., v.90, n.5, p.1244-1251, 2009. doi:10.3945/ajcn.2008.27327

TREPANOWSKI, J.F. et al. Effect of alternate-day fasting on weight loss, weight maintenance, and cardioprotection among metabolically healthy obese adults: a randomized clinical trial. JAMA, v.177, n.7, p. 930-938, 2017. doi: 10.1001/ jamainternmed.2017.0936

VARADY, K.A. et al. Effects of weight loss via high fat vs. low fat alternate day fasting diets on free fatty acid profiles. Scie. Reports, v.5, p.7561, 2015. doi: 10.1038/srep07561

YANG, W. et al. Alternate-day fasting protects the livers of mice against high-fat diet-induced inflammation associated with the suppression of Toll-like receptor 4/nuclear fator $\mathrm{\kappa B}$ signaling. Nutr. Res., v.36, n.6, p.586-593, 2016. doi: 10.1016/j. nutres.2016.02.001

ZIAEE, V. et al. The changes of metabolic profile and weight during Ramadan fasting. Singapore Med. J., v.47, n.5, p.409-414, 2006. 